\title{
Giant dermatofibroma - a rare clinical variant
}

\author{
Raj Kumar Meena ${ }^{1}$, Arambam Gautam ${ }^{2}$, A Mahendra Singh ${ }^{3}$, Sanjib \\ Waikhom ${ }^{4}$, I Ibomcha Singh ${ }^{3}$ \\ I (PGT, Department of Orthopaedics, Regional Institute Of Medical Sciences , Manipur , India) \\ ${ }^{2}$ (PGT, Department of Pathology, Regional Institute Of Medical Sciences, Manipur , India) \\ ${ }^{3}$ (Professor, Department of Orthopaedics, Regional Institute Of Medical Sciences, Manipur, India) \\ ${ }^{4}$ (Assistant Professor, Department of Orthopaedics, Regional Institute Of Medical Sciences, Manipur, \\ India)
}

\begin{abstract}
Dermatofibroma, also known as benign fibrous histiocytoma is a soft tissue tumor that usually occur in the mid adult life and shows a slight female predominance. Giant dermatofibroma, a very rare clinical variant is characterised by its unusually large size, benign biological behaviour despite its large size and same histopathological characterstics as conventional dermatofibroma .. We report a case of a 20 years old female presenting with a rapidly enlarging large mass in right leg in calf muscle region with history of progressive enlargement for past 4 years. The mass measured $13 \mathrm{~cm}$ in greatest dimension. The patient underwent total excision of the tumor with wide local margin and histopathological examination confirmed it to be Giant dermatofibroma. Microscopic picture showed fibroblastic proliferation with collagen production, histiocytes and storiform pattern and focal lymphocytic infiltrates. Regular follow up showed no recurrence. This case is reported due to its very rare occurance, academic interest and to differentiate giant dermatofibroma from other such conditions such as desmoid tumor and dermofibrosarcoma protubens .
\end{abstract}

Keywords: Dermatofibroma, Desmoid, Fibrous histiocytoma , Variant,

\section{Case Report}

A 20 years old female was admitted in the department of orthopaedics, RIMS hospital , Imphal with a swelling in the right leg in the calf muscle region for past 4 years. The swelling was asymptomatic but characterised by slow enlargement. The patient remembered no preceding episode of trauma or local irritation. There was no other significant or eventful past history.

On examination, a solitary swelling was observed at the mid calf muscle region of right leg,. It was $13 \mathrm{~cm} \times 10 \mathrm{~cm}$ in size, firm in consistency,non tender, non pulsatile and non mobile. There were no other appreciable swelling.

(figure 1)

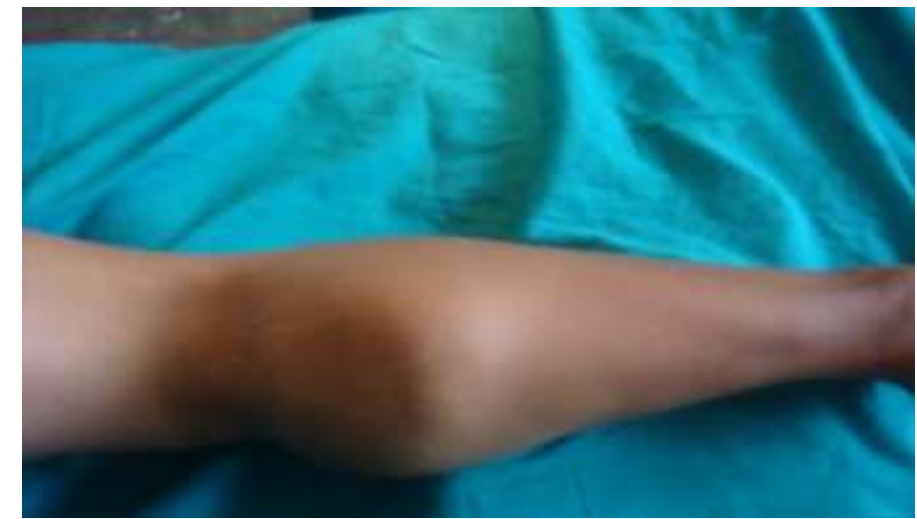

All routine investigations were within normal limits. Plain radiography ruled any bony involvement. Clinical examination showed soft tissue swelling. Surgical excision of the swelling was planned considering unusual large size and patient's apprehenshion due to cosmetic reasons.

Surgical excision of the tumor with wide local margin was done under spinal anesthesia. Intrapoeratively a well encapsulated greyish white mass was seen in the region of skin overlying calf muscle . It was seen to arise from dermal layer of skin extending till calf muscle. It was excised en bloc without damaging to any surrounding structures.

On histopathological examination , the gross picture was of globular soft tissue swelling measuring 13 $\mathrm{cm} \times 10 \mathrm{~cm}$ in greatest dimension, showing capsulated solid greyish firm area with intervening whitish bands (figure. 2) 


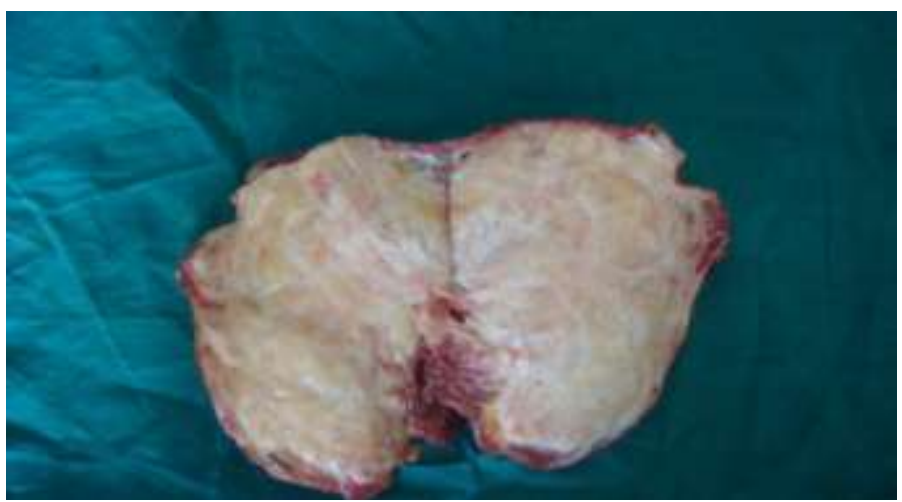

Microscopic picture showed fibroblastic proliferation with collagen production, epidermal hyperplasia, histiocytes and storiform pattern and focal lymphocytic infiltrates suggestive of benign dermatofibroma (benign fibrohistiocytic tumor). ( figure 3)

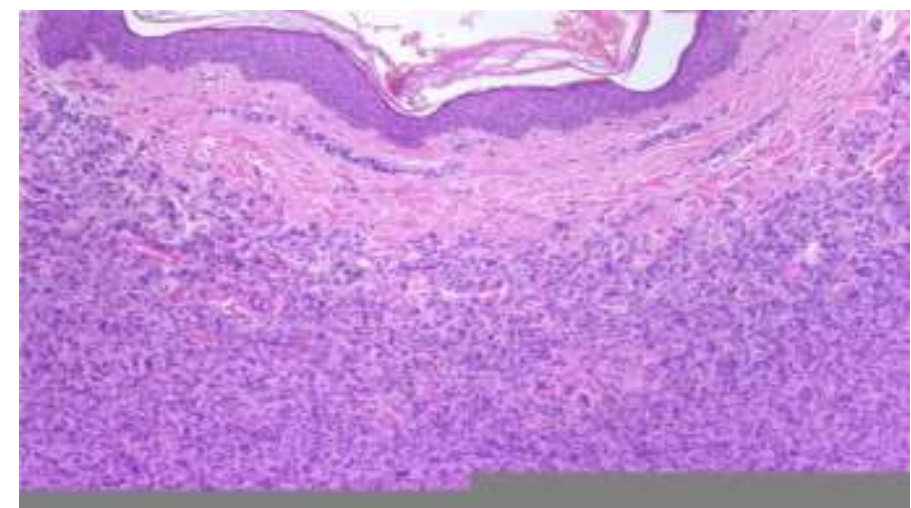

Post operatively recovery of the patient was uneventful and she was discharged after 10 days . Regular follow up was normal with no recurrence seen .

\section{Discussion}

Dermatofibroma is a common benign skin tumor that usually appear as slow growing nodule or papule in the dermis or subcutaneous tissue. ${ }^{1}$ The most frequent clinical presentation of dermatofibroma is of a red brown papule on the leg measuring a few millimetres in diameter, which is mostly asymptomatic .It almost invariably exhibit benign biologic behaviour . Pruritis and tenderness not uncommon. ${ }^{2}$.Dermatofibroma can occur at any age, but usually develops in young adulthood. Twenty percent of dermatofibroma occur in a person less than 17 years old .Male to female ratio is $1: 4 .^{3}$

Giant Dermatofibroma is a distinct but very rare clinical variety of dermatofibroma. When very large, the lesion may present clinically as Giant dermatofibroma . The features widely accepted as Giant Dermatofibroma are size $>5 \mathrm{~cm}$, benign biological behaviour despite its unusually large size and the same histopathological characterstics as conventional Dermatofibroma. ${ }^{4}$

The etiology of the lesion is controversial and thought to represent a reactive process or dermal response to injury following an insult such as folliculitis, arthropod bites or unspecified inflammatory conditions. $^{5}$

Histological findings in dermatofibroma consists of proliferation of spindle cells arranged in storiform pattern , epidermal hyperplasia , fibroblast proliferation with collagen production and infiltration of histiocytes .

The differential diagnosis of dermatofibroma must include dermatofibrosarcoma protubens and desmoids tumors .

In our case, the diagnosis of giant dermatofibroma was made mainly on the basis of clinical and histopathological findings. Considering its unusual large size , surgical excision with wide margin was done and on subsequent follow up no local recurrence was seen .

\section{Conclusion}

In our report, the diagnosis of giant dermatofibroma was made mainly based on clinical and histopathological findings. The speed of evolution and abnormally large size did raise a suspicion of malignancy. However histopathological examination provided the definite diagnosis of Benign Giant 
Dermatofibroma . Surgical excision with wide local margin appears to be the adequate treatment modality as regular follow up was not associated with any reccurance. This case is reported because of its rarity in occurance , to differentiate it from other soft tissue tumors such such as dermatofibrosarcoma protubens and desmoids tumor and academic interest .

\section{Acknowledgment}

The authors would like to thank the patient for her kind permission to report this case.

\section{References}

[1] Kamino H , Salcedo E. Histopathologic and Immunohistochemical diagnosis of benign and malignant fibrohistiocytic tumors of the skin . Dermatol Clin 1999;17:487-505

[2] Rapini R P .Practical Dermatopathology .Philadelphia. Mosby ;2005

[3] Vali R G , Valia A R . IADVL Textbook Of Dermatology . $3^{\text {rd }}$ ed. Mumbai:Bhalani publishing House ;2006

[4] Requena L, Farina M C, Fuente C, Pique E, Olivas M, Murtin L:Giant dermatofibroma - a little known clinical variant of dermatofibroma :J Am Acad Dermatol 1994 May;30(5):714-718

[5] Hueso L, Jimenez O S, Rubio A A . Giant dermatofibroma : Case report and review of literature :Actus Dermo-Sifiliograficus 2007 ;98(2):121-124

[6] Rosenberg A E .Bones, Joints and Soft Tumors. In: Kumar V ,Abbas A K, Fausto N, Editors . Robbins and Cotrans Pathologic Basis Of Disease $7^{\text {th }}$ ed .Philadelphia :Saunders ;2004. P .1320-1321. 